\title{
Techno-economic evaluation of load activation quotas as a concept for flexible load management
}

\author{
Nicolas Thie ${ }^{1} \bowtie$, Simon Koopmann ${ }^{1}$, Armin Schnettler ${ }^{1}$, \\ Sascha Hillenbrand ${ }^{2}$, Andreas Kopp ${ }^{2}$ \\ ${ }^{1}$ Institute for High Voltage Technology (IFHT), RWTH Aachen University, Aachen, Germany \\ ${ }^{2}$ Technik Innovation (TI), Netze BW GmbH, Stuttgart, Germany \\ $\bowtie$ E-mail: thie@ifht.rwth-aachen.de
}

\begin{abstract}
Flexible load management concepts are increasingly relevant due to a rising share of flexibilities at distribution grid level, e.g. power-to-heat systems, electric vehicles or home battery storages. In a market oriented load management, uniform price signals can lead to higher load simultaneity factors and grid congestions. Therefore, load management concepts have to consider the prevention of grid congestions, e.g. by load activation quotas. The quota represents the maximum share of flexible loads per grid segment for each point in time that can be activated without causing grid congestions. Grid expansion measures increase the possible activation quota and lead to higher flexibility in operation. For an aggregator of flexible loads, grid expansion increases the potential for procurement cost reduction. On the other side, it causes capital costs for the distribution system operator. This study introduces a methodology for the techno-economic evaluation of LAQ as a concept for flexible load management in future distribution grids, which is applied to an exemplary medium-voltage grid in Southern Germany. The analysis considers both the investment costs for grid expansion by the grid operator and operational savings in procurement due to an increased flexibility for an aggregator. The cost-efficient trade-off from a micro-economic perspective is determined.
\end{abstract}

\section{Introduction}

In course of the energy transition, new kinds of flexibilities are introduced into the power system. Especially the share of power-to-heat systems $(\mathrm{P} 2 \mathrm{H})$ and electric vehicles $(\mathrm{EV})$ is expected to increase [1]. Additionally, the installation of home battery systems to support self-consumption of photovoltaic systems becomes more economically attractive [2]. At the same time, the rising share of intermittent generation leads to high generation peaks in the power system causing volatile energy prices. For an aggregator, these new types of units with thermal or electrical storage capacities provide flexibilities to shift consumption to times of high renewable generation and to exploit low energy prices.

However, $\mathrm{P} 2 \mathrm{H}$ are currently operated in load groups with static activation periods. These load groups significantly limit activation times and do not exploit the full flexibility potential [3].

A market-oriented load management could increase flexibility and therefore reduce energy costs for flexible loads. On the downside, market-oriented load management causes higher simultaneity factors due to the uniform price signals for all flexible loads. Hence, the risk of grid congestions at distribution level is increased [4].

Load activation quotas (LAQ) as a concept for load management consider the prevention of grid congestions. The quota limits the share of flexible loads in a grid segment for each point in time that can be activated without causing grid congestions [3]. Investments in grid expansion increase the flexibility as well as the opportunities to reduce energy procurement costs for market-oriented aggregators, but cause capital costs for the grid operator.

In this paper, a methodology to evaluate the economic efficiency of LAQ is presented. The savings in energy costs due to flexibility in load management on the one hand are confronted with grid expansion costs to increase flexibility on the other hand. The objective of the presented method is the quantitative analysis of the conflicting objectives and the determination of the cost-efficient trade-off between increased flexibility and grid expansion. Various grid expansion levels are defined which cover the range of possible grid expansion measures (Fig. 1). For the respective expansion levels, the energy procurement costs as well as grid investment costs are evaluated.

\section{Load activation quotas (LAQ)}

The concept of LAQ for grid compatible load management is based on the definition in [3]. LAQ are calculated for various grid layers in a $15 \mathrm{~min}$ interval. The defined grid layers for a medium-voltage grid are shown in Fig. 2. In this paper, P2H systems, EV and home battery systems are considered as flexible loads. In general all types of loads that offer flexibility for load shifting can be included in the method. The quota is calculated by the distribution system operator (DSO) on the basis of knowledge about the grid topology, installed loads and generators. The line and transformer utilisation as well as the maximum voltage band deviations are considered as grid restrictions. The DSO communicates the results (Fig. 3) to the respective utilities or aggregators, which can optimise the energy procurement of their flexible loads within the restrictions of the LAQ.

The algorithm for the determination of LAQ is based on an iterative process, which uses power flow calculations and power flow sensitivities. The algorithm starts with a complete activation of all flexible loads. Loads, which can affect the existing grid congestions are reduced iteratively until all congestions in the grid are eliminated. The effect of the individual loads on the existing congestions is determined by the power flow sensitivities. 


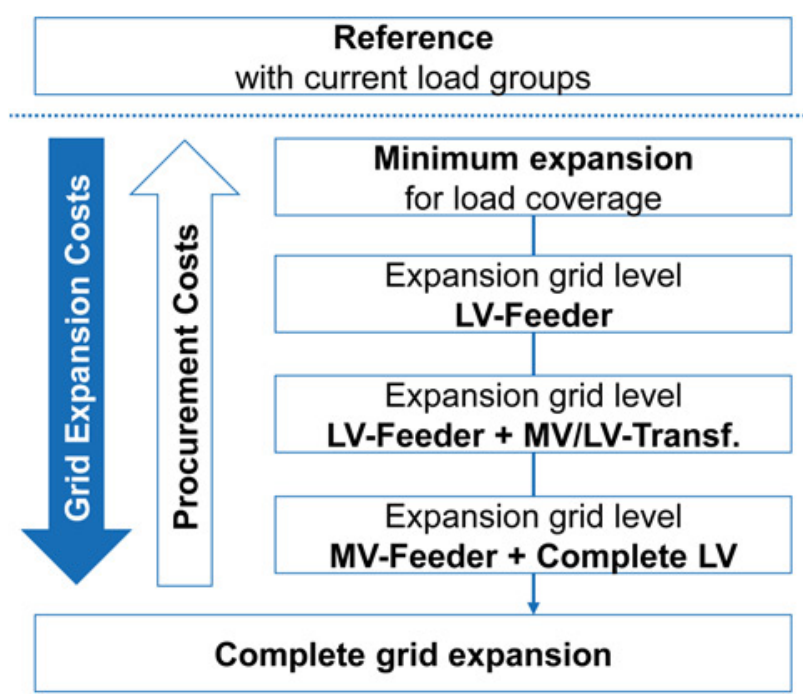

Fig. 1 Trade-off between grid expansion and procurement costs

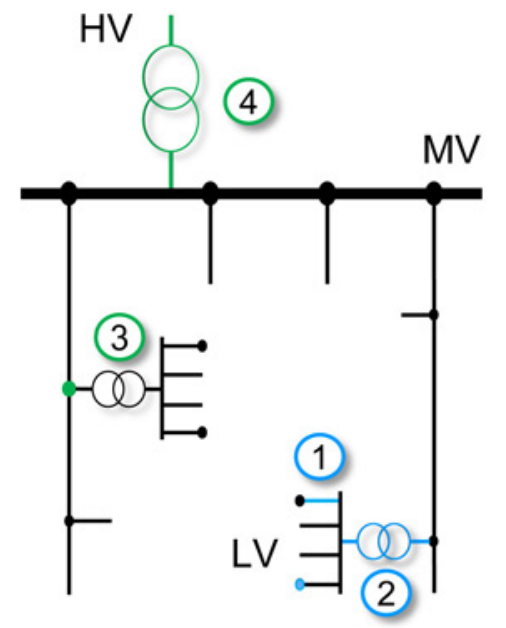

(4) HV/MVTransformer: Transformerutilization

(3) MV-Node: Line-utilization Voltage band

(2) $\mathbf{M V} / \mathbf{L V}$ Transformer: Transformerutilization

(1) LV-Feeder: Line-utilization Voltage band

Fig. 2 Defined grid levels for $L A Q$

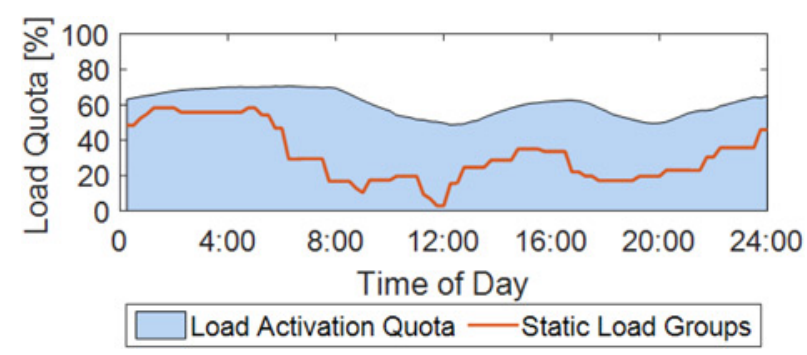

Fig. 3 Load activation quota and static load groups

\section{Evaluation methodology}

The evaluation method to determine the efficient trade-off between grid investment costs and energy procurement costs for flexible loads includes various model steps (Fig. 4).

In addition to the algorithm for the calculation of LAQ, the method includes a heuristic grid expansion model to determine investment costs as well as a linear optimisation model to minimise procurement costs. The evaluation is carried out on the basis of forecasts for future penetration rates of flexible loads and renewable generation in the distribution grid under consideration. The evaluation results include grid expansion costs and

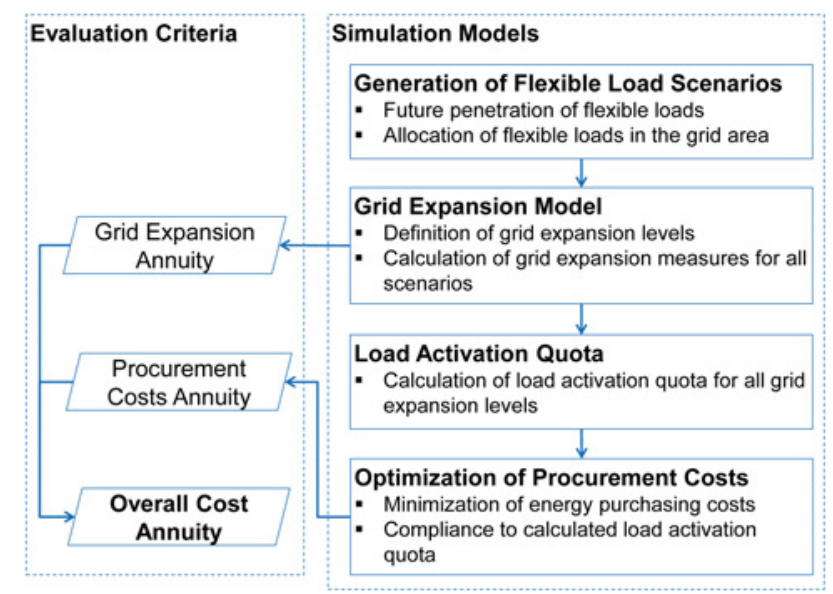

Fig. 4 Evaluation method overview

Table 1 Distribution scenarios for flexible loads

\begin{tabular}{lc}
\hline Scenario & Concentration of flexible loads \\
\hline $\begin{array}{l}\text { worst } \\
\text { medium } \\
\text { best }\end{array}$ & $\begin{array}{c}\text { in highly utilised LV grids } \\
\text { uniformly distributed } \\
\text { in lowly utilised LV grids }\end{array}$ \\
\hline
\end{tabular}

procurement costs, which are transformed into annuities and combined to overall costs to determine the cost-efficient trade-off.

\subsection{Generation of flexible load scenarios}

The flexible load scenarios are defined based on forecasts for future development of the aggregated flexible load capacity as well as average unit sizing per customer. The forecasts are provided by the local DSO Netze BW GmbH [5] as well as public studies [1, 6]. To simulate the actual supply task, the aggregated forecasts have to be distributed among the customers in the grid area. To investigate multiple supply tasks, the concentration of flexible loads in the grid is varied in the form of three distribution scenarios (Table 1). For example, in the scenario 'Worst' households in highly utilised low-voltage (LV) grids receive a high probability for the allocation of flexible loads. The individual units (flexible loads and generation) are allocated iteratively until the forecasts for future penetration are met.

\subsection{Grid expansion model}

The defined grid expansion levels serve as inputs for the grid expansion model. The minimum expansion includes only expansion measures, which are needed to cover consumption of all flexible loads. Additional flexibility for market oriented load shifting or a simultaneous activation is not considered as cause for grid expansion. Hence, this level constitutes the minimum regarding investment costs. Complete expansion on the other hand enables full flexibility for market-oriented load management. Therefore, a $100 \%$ simultaneity factor at all times is possible, which implies the maximum potential for the reduction of procurement costs. The different layers range from LV line, medium-voltage (MV)/LV substation and MV line up to the HV/ MV substation. Additionally, a reference is defined by assuming todays German planning principles for distribution grids ([7]) and load groups without the use of LAQ.

3.2.1 Definition of minimum grid expansion: The minimum expansion is determined based on the minimum requirements for supplying all flexible loads. This implies minimum requirements regarding the consumption, power and activation periods, i.e. in 

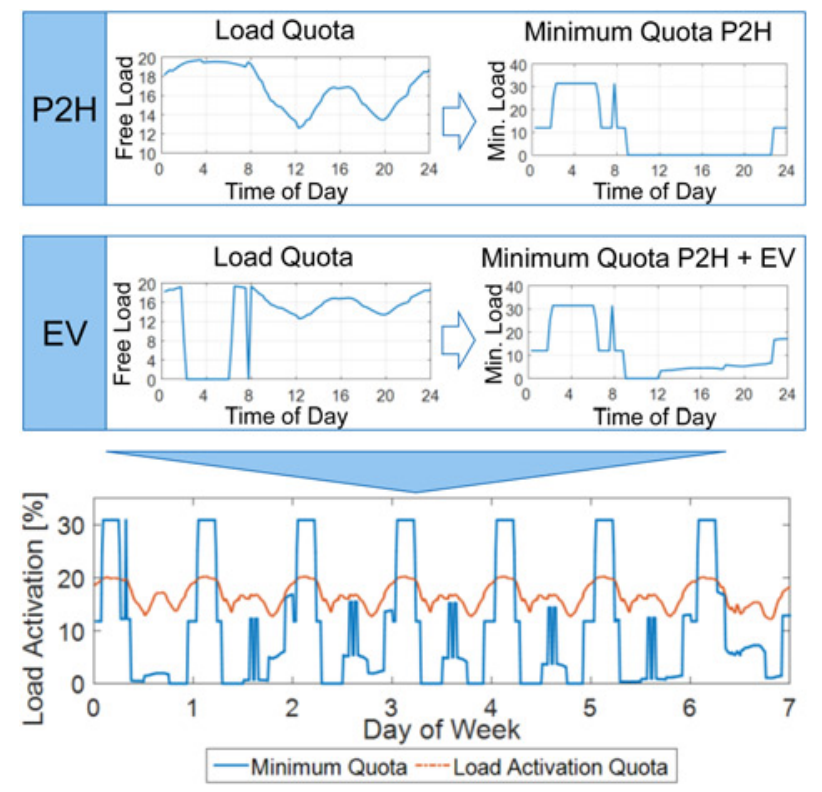

Fig. 5 Determination of the minimum quota

form of a minimum LAQ. The minimum quota only applies for P2H systems and $\mathrm{EV}$, because a minimum consumption is not required for home battery systems. The daily consumption is calculated based on representative load profiles. For $\mathrm{P} 2 \mathrm{H}$ systems the additional requirement of binary activation applies, because activation with partial load is not possible [5]. For EV, usage limitations for drivers have to be prevented. Therefore, the minimum quota has to allow sufficient charging in the limited period, when the EV is connected to the grid. This time period is implemented as an adjustable parameter of the model and is set to $6 \mathrm{~h}$ for this investigation, e.g. in the night or during work.

For each grid level, the minimum quota is calculated by aggregating the minimum load activation of all connected $\mathrm{P} 2 \mathrm{H}$ systems and EVs.

The activation of the individual units is determined with a heuristic approach, which distributes the load activation as efficiently as possible considering existing grid capacities. Basis of the heuristic approach is the calculated LAQ. The load activation, beginning with the unit with the highest load, is placed in the time with the highest LAQ. The unit is activated until the consumption is covered. Grid expansion is only needed, if the minimum quota in a grid segment exceeds the possible LAQ (Fig. 5).

3.2.2 Grid expansion heuristic: In the grid expansion heuristic, the expansion measures and the associated investment costs are calculated for the defined expansion levels (Fig. 6).

The years 2020 and 2030 are calculated hierarchically. Therefore, expansion in 2030 is based on the grid model including expansion measures executed in 2020. The necessary grid expansion is determined via power flow calculations. Aside from the grid model, the heuristic uses profiles for passive load and generation as input parameters. The load profiles for flexible loads represent the targeted degree of flexibility in the respective grid expansion level (Table 2).

Due to the different load profiles, the resulting grid model includes all grid expansion measures necessary to allow the targeted flexibility. For the expansion, standard grid equipment and costs are assumed [5].

\subsection{Optimisation of aggregator procurement costs}

The procurement optimisation calculates the possible savings in energy purchases on the energy market for the respective grid expansion levels. The calculation is carried out using a mixed integer linear programming model, which minimises the product of

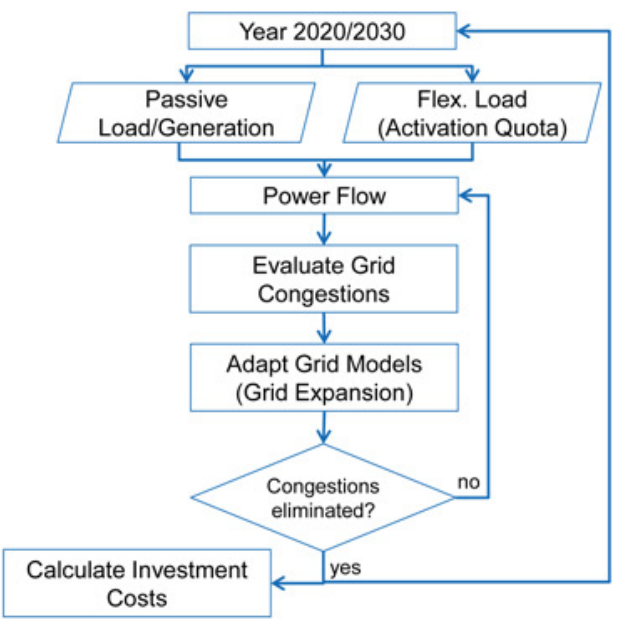

Fig. 6 Grid expansion heuristic

Table 2 Load profiles depending on expansion level

\begin{tabular}{lc}
\hline Expansion level & Load profile \\
\hline $\begin{array}{l}\text { reference } \\
\text { minimum }\end{array}$ & static activation periods \\
layer wise & minimum quota \\
complete & layer wise full flexibility \\
& complete flexibility on all layers \\
\hline
\end{tabular}

Table 3 Characteristics of the price forecast 2025

\begin{tabular}{lc}
\hline Parameter & $€ / \mathrm{MWh}$ \\
\hline maximum price & 105 \\
minimum price & -7 \\
average price & 43 \\
standard deviation & 15 \\
\hline
\end{tabular}

market prices $c_{\text {Spot }}$ and amount $x_{\text {Spot }}$ on the energy market for all flexible load units $(n)$ and all time steps $(t)$ (1). Technical restrictions of the flexible loads are introduced as optimisation constraints, e.g. maximum storage and charging capacities. A detailed description of the optimisation model is presented in [8]. LAQ are integrated as a new set of constraints (2) and limit the maximum load activation in every time step. Hence, these constraints guarantee the optimisations' compliance with the grid restrictions.

$$
\begin{gathered}
\min \sum_{n=1}^{N} \sum_{t=1}^{T} c_{t, \text { Spot }}^{n} \cdot x_{t, \text { Spot }}^{n} \\
x_{t, \text { Spot }}^{n} \leq \mathrm{LAQ}_{t}^{n} \quad \forall t \in T, n \in N
\end{gathered}
$$

The optimisation is carried out for a full year. To consider future developments of energy prices, a price forecast for 2025 is used (Table 3). The price forecast results from a simulation of the European energy market, which is based on the network development plan 2015 scenario 2025B $[9,10]$.

\section{Introduction of the model region}

The grid area 'Boxberg' includes a $20 \mathrm{kV}$ MV grid with 72 underlying LV grids and is connected to the HV grid via a 25 MVA transformer. The load reaches a maximum peak of 16.2 MW. Already today, the region is characterised by a high share of $\mathrm{P} 2 \mathrm{H}$ systems and renewable generation with a capacity of 11.5 MW and 24.3 MW, respectively. The expected increase of 


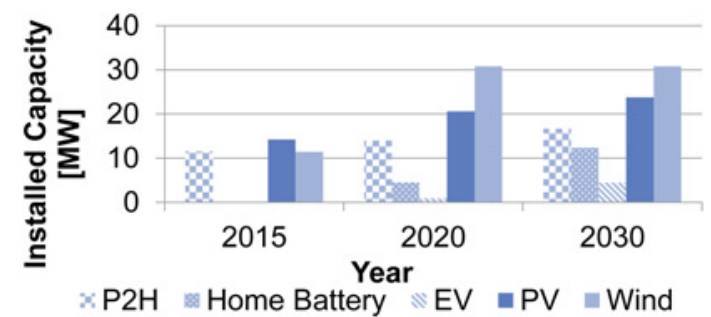

Fig. 7 Load and generation forecast until 2030

flexible loads and renewable generation until 2030 is summarised in Fig. $7[1,5]$. The installed flexible loads accumulate to about 19.4 MW in 2020 and 33.5 MW in 2030.

\section{Evaluation results}

The evaluation method is applied to the model region 'Boxberg'. The analysis shows annuity based

- grid expansion costs,

- procurement costs and,

- overall costs.

Based on the overall costs for the grid expansion layers, the efficient trade-off can be determined. The analysis is carried out for all three-distribution scenarios presented in Table 1 .

Prior to the cost analysis, the effect of grid expansion measures on the LAQ is analysed. Fig. 8 shows grid expansion costs and average LAQ for the defined grid expansion levels in the scenario Medium 2020. Aside from reference and minimum expansion, the layer wise expansion is presented. In single-layer expansion levels, expansion measures are limited to only one grid layer (e.g. LV feeder). Multi-layer levels include multiple grid layers (e.g. LV + MV grid) up to complete expansion.

The analysis shows a low effect on the LAQ and high investment costs for layer wise expansion in comparison to minimum expansion. The level LV + MV, including expansion of the LV grid and MV lines only, increases the LAQ by $4 \%$ compared to minimum expansion because the HV/MV transformer remains a bottleneck and limits the load activation. Therefore, only a complete expansion significantly increases the LAQ. Regarding investment costs, especially an expansion of the MV grid causes high costs (cf. MV feeder and LV + MV). Furthermore, investment in cables (LV feeder) is significantly more expensive than upgrading transformers (MV/LV). In the LV grid this difference in costs is caused by the low price of standard MV/LV transformers. In the MV grid, cables have a length in the range of kilometers, which causes high costs if several lines have to be replaced.

Based on these initial results, the full cost analysis is shown for the expansion levels reference, minimum and complete expansion (Fig. 9). Additionally, all three-distribution scenarios are shown for the respective years 2020 and 2030 .

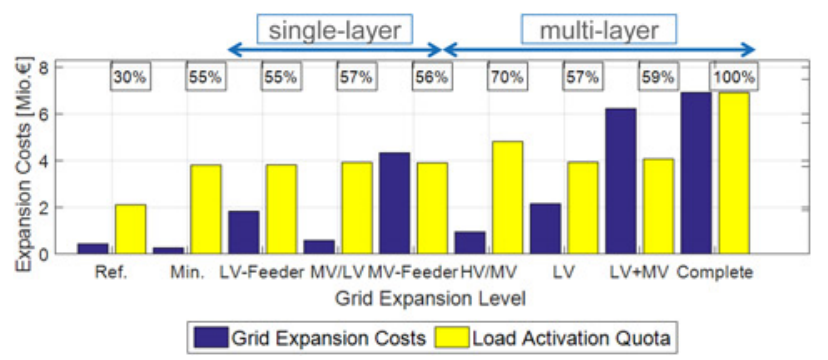

Fig. 8 Effect of grid expansion on the $L A Q$
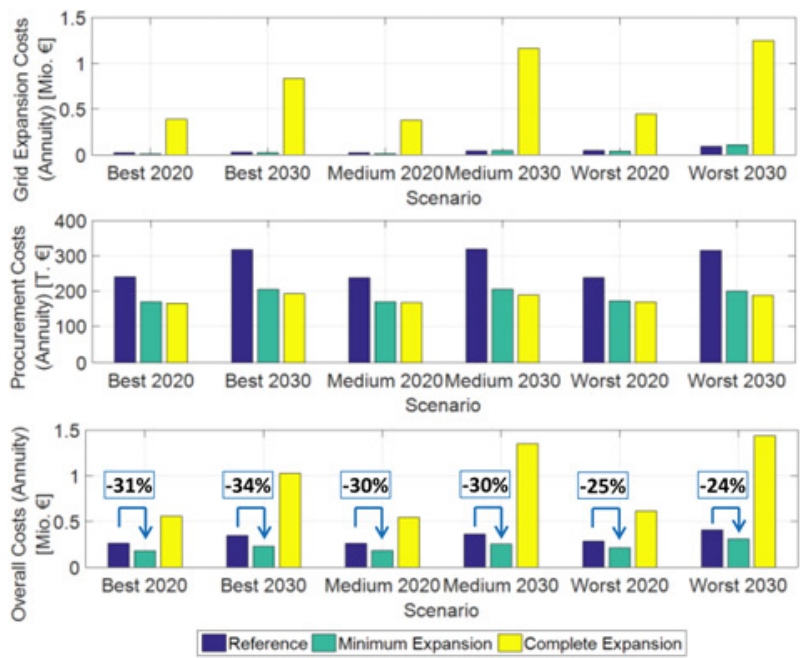

Fig. 9 Cost comparison depending on grid expansion

\subsection{Expansion costs}

The expansion cost annuities amount to about 100,000-200,000 € for reference and minimum expansion and do not change significantly over the distribution scenarios (Fig. 9). Complete expansion on the other hand causes a multiple of these annual costs with approximately $500,000 €$ in 2020 and over 1 million $€$ in 2030. The large difference between reference and minimum expansion on the one hand and complete expansion on the other hand is caused by the $100 \%$ flexibility requirement in case of complete expansion, which represents the benchmark for the theoretic maximum of grid expansion costs. This means, the grid has to be planned for a simultaneity factor of $100 \%$ for all flexible loads, which causes a significant need for grid expansion. The load groups used in the reference case reduce the simultaneity factor because the activation periods differ in the respective load groups. In the minimum expansion, activation times are even optimised to minimise the need for grid expansion.

\subsection{Procurement costs}

Procurement costs show a significant advantage of LAQ even in the case minimum expansion compared to the use of load groups in reference. Due to the higher flexibility in load activation, costs can be reduced by about $30 \%$. Complete expansion only slightly improves the cost reduction. These results show that a high share of the possible cost reduction can be reached based on the flexibility in the minimum expansion. Further investment in grid expansion does not achieve procurement cost reductions to the same extent.

\subsection{Overall costs}

For the investigated model area, the case minimum expansion causes minimal overall costs and represents the efficient trade-off solution between grid expansion and procurement costs. Overall costs can be reduced by $24-34 \%$ compared to the reference case. This cost advantage is constant over all distribution scenarios and the investigated time horizon. Due to the high demand for grid expansion, costs of complete expansion exceed the overall costs of minimal expansion by a multiple.

\section{Summary and outlook}

In this paper, a techno-economic evaluation method for LAQ as a grid compatible load management concept has been presented and 
applied to a model region in Southern Germany. LAQ limit market-oriented load management under consideration of grid restrictions. Therefore, a conflict of objectives exists between grid expansion and cost reductions on the energy markets. The presented method determines the cost-efficient trade-off solution. For the investigated model region, a minimum grid expansion in conjunction with the use of LAQ represents this trade-off and allows a cost reduction of $24-34 \%$ compared to the reference scenario. The use of LAQ increases flexibility compared to the currently used static activation periods. Hence, this flexibility allows an optimisation of procurement costs. Even in case of minimum grid expansion, a significant cost reduction can be realised. Additionally, the minimum grid expansion causes the lowest investment costs.

The results shown in this paper only apply to the investigated model area. For a more representative analysis, the presented method will be transferred to the whole network area of Netze BW via the evaluation of representative MV and LV grids. The goal is a verification of the determined cost-efficient trade-off and the economic efficiency of LAQ.

\section{References}

1 Schlesinger, M.: 'Entwicklung der Energiemärkte - Energiereferenzprognose' (Prognos, Berlin, Germany, 2014)

2 Kairies, K.: 'Wissenschaftliches mess- und evaluierungsprogramm solarstromspeicher', BMWi, 2016

3 Koopmann, S.: 'Grid compatible load management of power-to-heat systems based on load activation quotas', ETG, 2015

4 Hallberg, P.: 'Active distribution system management - A key tool for smooth integration of distributed generation', Eurelectric, 2013

5 Internal Netze BW documentation

6 Huppertz, P.: 'Stabilizing effects by means of low-power heat pumps in electrical low-voltage grids', VDE, 2015

Nagel, H.: 'Systematische netzplanung', 2008

8 Koopmann, S.: 'Planung von verteilungs-netzen unter berücksichtigung von flexibilitäts-optionen', RWTH Aachen University, 2016

9 Raths, S.: 'The energy system development plan (ESDP)', ETG, 2015

10 Netzentwicklungsplan Strom 2025, 2015 